\title{
Could periodic patterns in human mortality be sensitive to solar activity?
}

\author{
R. Díaz-Sandoval ${ }^{1}$, R. Erdélyi $^{1}$, and R. Maheswaran ${ }^{2}$ \\ ${ }^{1}$ Solar Physics and Space Plasma Research Centre, School of Mathematics and Statistics, University of Sheffield, \\ Hicks Building, Hounsfield Rd, Sheffield S3 7RH, UK \\ ${ }^{2}$ Public Health GIS Unit, School of Health and Related Research, University of Sheffield, Regent Court, 30 Regent St, \\ Sheffield S1 4DA, UK
}

Received: 31 December 2010 - Revised: 11 March 2011 - Accepted: 18 March 2011 - Published: 25 June 2011

\begin{abstract}
Seasonal behaviour of human diseases have been observed and reported in the literature for years. Although the Sun plays an essential role in the origin and evolution of life on Earth, it is barely taken into account in biological processes for the development of a specific disease. Higher mortality rates occur during the winter season in the Northern Hemisphere for several diseases, particularly diseases of the cardiovascular and respiratory systems. This increment has been associated with seasonal and social causes. However, is there more behind these correlations, in particular in terms of solar variability? In this paper we attempt to make a first step towards answering this question. A detailed wavelet analysis of periodicities for diseases from England and Wales seem to reveal that mortality periodicities ( 3 days to half a year) could be due to the Earth's position around the Sun. Moreover, crosswavelet and wavelet coherence analysis show common features between medical diseases and solar proxies around solar maximum activity suggesting that this relation, if any, has to be searched in times of high solar activity.
\end{abstract}

Keywords. Electromagnetics (Biological effects) - Geomagnetism and paleomagnetism (Biomagnetism; Time variations, diurnal to secular)

\section{Introduction}

Periodic behaviour in mortality is reported elsewhere in the literature (e.g., Healy, 2003). The annual increment at the end of the year in cardiac, mental and asthma diseases is well known and has been often associated with weather conditions (Weiss, 1990; Sheth et al., 1999). These increments have also

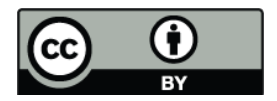

Correspondence to: R. Díaz-Sandoval (r.diaz@sheffield.ac.uk) been related to the social behaviour of humans during holidays and close to Christmas and New Year time (Phillips et al., 2004). Moreover, there are some claims (Breus et al., 2008) that propose solar activity as an environmental condition for the resonant response of human diseases. Solar activity is changing all the time; following well known periodic patterns that reach a minimum approximately every 11 years, passing for periods of maximum activity. The most studied feature of this activity has been the sunspots, which have been registered on a daily basis since 1818 (NOAA Solar Data Services website). Transient phenomena as solar flares and coronal mass ejections are more common during periods of high activity and propagate along the interplanetary medium (see Kane, 2005, for a review) reaching the planets of the solar system and even screening the arrival of cosmic rays coming from galactic sources. Depending on the electromagnetic conditions of the Earth's magnetosphere, some of these transients can affect the Earth's environment causing geomagnetic storms, auroras or changes in the electronic content of the ionosphere (Kane, 2005). Solar transient and cosmic ray behaviour has also been related with electric atmospheric conditions such as lightning (Schlegel et al., 2001) and Schumann resonances (Sátori et al., 2005) which have periodic components similar to brain wave frequencies (Direnfeld, 1983). Some hypotheses suggest that these atmospheric resonances could affect the human nervous system and as a consequence alter the human being homoeostasis causing or contributing to the development of a particular disease in weak biologic systems (Cherry, 2002).

Following the complex puzzle that proposes a response of the human being to the solar activity behaviour, many statistical works (see Cornélissen et al., 2002; Palmer et al., 2006; Breus et al., 2008, for reviews), experiments (e.g., Bureau and Persinger, 1995; Babayev and Allahverdiyeva, 2007), and theoretical models (e.g., Panagopoulos et al., 2002; DíazSandoval and Mendoza, 2004) have been carried out over the

Published by Copernicus Publications on behalf of the European Geosciences Union. 
years in order to elucidate this controversial conjecture. Annual, weekly, and semi-weekly periodicities have been found in cardiac diseases from diverse databases (Cornélissen et al., 2002; Díaz-Sandoval et al., 2008, and references therein) and also in geomagnetic proxies. Moreover, cardiac and mental diseases show statistical relations with solar cycle, Ap geomagnetic index, and sudden decrements (Forbush decreases) in cosmic ray counts (Palmer et al., 2006, and references therein) in ground-based monitors. Electroencephalographic patterns present alterations during severe geomagnetic storms (Babayev and Allahverdiyeva, 2007). Cell ions and proteins show periodic behaviour in the presence of magnetic fields (Ulmer, 2002) and recently, there are hypotheses that ultraviolet radiation coming from the Sun in different solar activity stages could be a main factor for the development of human immune system (Davis Jr. and Lowell, 2006).

In this paper we analyse periodicities of medical data from England and Wales covering most of Solar Cycle 23. Using wavelets (Torrence and Compo, 1998), a recent mathematical tool developed to study the periodic behaviour of nonstationary series in the time-frequency space, we explore periodic patterns in medical data during the months when the Earth's relative position is close and far away from the Sun. We found a higher content of high-frequency periodicities (above the 95\% confidence level for wavelet scale average) when the Earth is closer to the Sun. This occurs mostly for short period bands for $88.9 \%$ of the cases; in fact, $77.8 \%$ of them show increases larger than 1.5 times. Moreover, we determine the level of coherence between periodicities of medical series and solar/geomagnetic activity proxies using cross wavelet and wavelet coherence analysis (Grinsted et al., 2004), finding a high coherence of annual and semi-annual frequencies when the sunspots and AA and Ap geomagnetic indices are close to their maxima.

\section{Data and methodology}

Medical data was obtained from the Office for National Statistics, United Kingdom for England and Wales and consisted in dataset of the number of daily mortality for the period dating from 1 January 1997 to 31 December 2005 (3287 days) for the following diseases: 409984 cases of Heart attack known as Myocardial Infarction Disease (MID); 450011 cases of Cerebrovascular Disease (CVD); 82412 cases of Heart Failure (HF); and 10877 cases of Asthma. MID is split in three age groups: 0-64 years (58 248 cases), 65-74 years (95206 cases) and over 75 years (256530 cases). CVD has the same division groups: 0-64 years (23 721 cases), 65-74 years (57948 cases) and over 75 years (368 342 cases). Asthma is split in two age groups: 0-74 years (5579 cases) and over 75 years (5298 cases). HF is not split.

Based on previous studies about the analysis of the relationship between solar activity and medical diseases (Palmer et al., 2006, and references therein), sunspot, AA and Ap geomagnetic indices were chosen. The sunspots daily number is a widely used proxy of solar activity (Kane, 2005). AA geomagnetic index is a proxy of daily global geomagnetic activity measured for two antipodal stations and the Ap geomagnetic index is a proxy of daily global geomagnetic activity averaged for twelve stations (Mayaud, 1980). The three of them were obtained from the National Oceanic and Atmospheric Administration (NOAA), USA database (http://www.ngdc.noaa.gov/stp/SOLAR) and cover the same period as the medical diseases series (from 1 January 1997 to 31 December 2005). The studied time period began sixth months after the solar minimum of Solar Cycle 23 that occurred in May 1996 (Kane, 2001). On the other hand, sunspot maximum took place on March 2000 (Kane, 2001). The solar minimum of Solar Cycle 24 has not yet fully determined at the present due to a recent long and unusual period of low solar activity. AA and Ap indices reached their maximum values on 11 May $2003(381.25 \gamma)$ and 19 June $2003(246 \gamma)$, respectively (numbers extracted from NOAA databases).

For medical and heliogeophysical data, we performed a time behaviour analysis and an analysis of frequencies using the Fast Fourier Transform (Press, 1992) and the Wavelets Transform methods (Torrence and Compo, 1998; Grinsted et al., 2004). The Fast Fourier Transform (FFT) computes the Discrete Fourier Transform, which extracts the periodic components of a time series using sinus and cosines basis (Press, 1992). The obtained plot is a variation of power spectral density as a function of frequency until a certain limit value, called the Nyquist frequency which is equal to $1 /(2 \Delta t)$ where $\Delta t$ is the sampling time. The FFT spectrum gives information about frequency content in a time series within a given period of time. In order to determine significant peaks, we used a $t$-test following the methodology explained by Jenkins and Watts (1967). However, for non-stationary series, FFT does not give enough information about time evolution of frequency peaks but only gives a global spectrum of the periodicities. On the other hand, wavelet transform is capable of analysing variations of power in the time-frequency space. It means that the wavelet transform may extract frequency information from a signal in a defined period of time. Assuming a time series $x_{n}$ with equal time spacing $\Delta t$, the continuous wavelet transform is defined as the convolution of $x_{n}$ with a wavelet function called "mother wavelet" that depends on two parameters, time and scale. By varying the scale and translating the wavelet in time, it is possible to construct a rather complete picture of periodic features in the time-frequency space (Torrence and Compo, 1998).

After characterising the time and frequency behaviour of medical and heliogeophysical data in a separate way, we performed wavelet coherence analysis in order to obtain the relation between the periodicities of both types of series. These analyses were cross correlation and cross wavelet analyses. Cross wavelet and wavelet coherence are analyses developed 

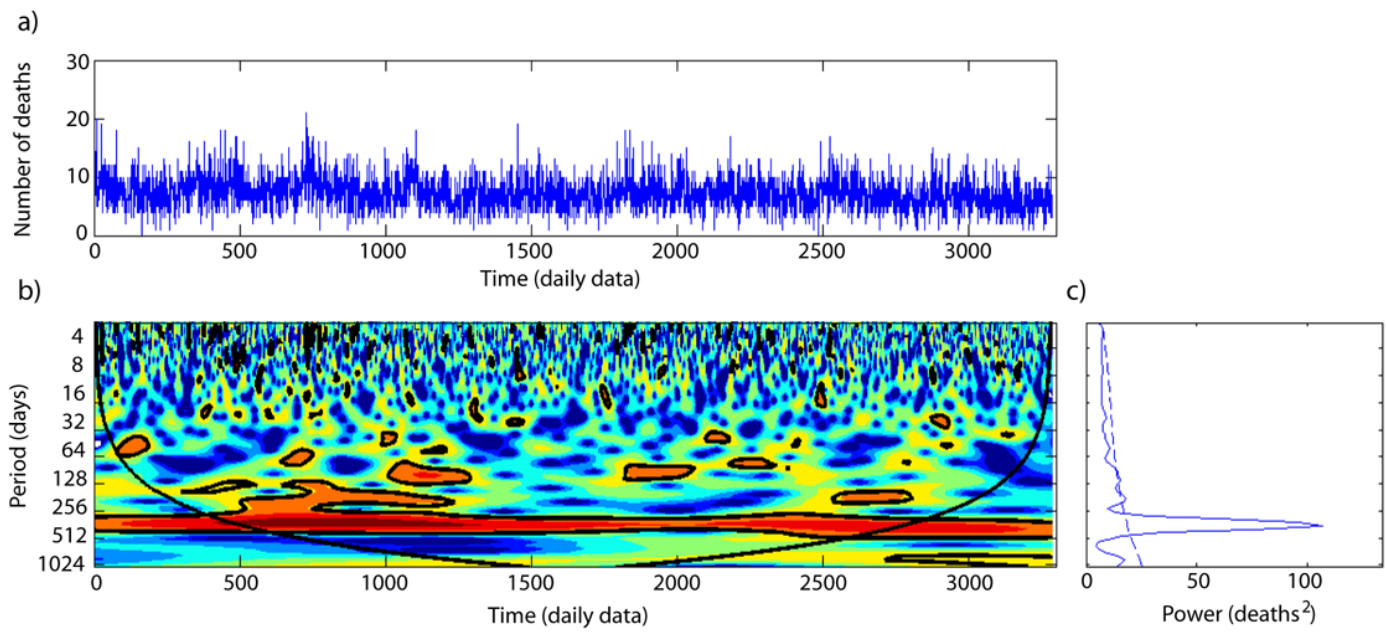

Fig. 1. Wavelet analysis for CVD 0-64 years group. (a) Time series. (b) Power wavelet spectrum. (c) Global wavelet spectrum. (d) Scale average time series for the following frequency bands: 3-4, 6-8, 12-16, 25-32, 32-64, 64-128, 128-256, 330-400, 500-600, 601-700 days.

to determine the level of coherence of two time series based on the wavelet transform (Grinsted et al., 2004). The cross wavelet transform shows the common power and the relative phase of two series in time-frequency space. This is an initial measurement of the level of relation between two series in time-frequency space. Wavelet coherence is analogous to cross wavelet analysis but introduces a smoothing operator in order to localize all possible common power and relative phase of the two series and not only the higher common power. These two analyses are used in a complementary way here.

\section{Time trends of diseases}

All MID groups and CVD 65-74 show a decreasing trend of the number of deaths that could be related with reductions of risk factors for these diseases in England and Wales (Unal et al., 2004). MID, CVD and HF show a clear annual behaviour with maxima at the end (start) of every year (e.g. see Fig. 1 for the case of CVD 0-64). For most of the diseases, maximum values occurred at the beginning of 1997. After performing a year-superposed analysis (Chapman and Bartels, 1962) for every group removing the 29 February for leap years (2000 and 2004), the yearly behaviour pattern is clearly recognisable in all the diseases, even for Asthma groups. However, for CVD 0-64, maximum values take place during January or December while minimum values occur for summer months.

\section{Frequency behaviour of diseases}

In an FFT analysis of the raw data we found the principal periodicities of each series. There are low frequency peaks in all diseases corresponding mainly to $\sim 1$ year ( \pm 50 days),
0.5 year ( \pm 6 days for most of them) and $120 \pm 4$ days (except MID 0-64), as we expected from the time series behaviour. There are conspicuous peaks of $1.6 \pm 0.3$ years for MID $\geq 75$ and CVD 0-64, 1.6 \pm 0.4 years for $\mathrm{HF}$ and $1.6 \pm 0.2$ years for Asthma $0-74 ; 1.4 \pm 0.4$ years for CVD 65-74 and Asthma $\geq 75$ years, $1.4 \pm 0.6$ years for $C V D \geq 75$ years; and $1.2 \pm$ 0.2 years for Asthma 0-74. At high frequencies, the presence of peaks was only evident for Asthma groups with $4.1 \pm 0.1$ days the highest peak for Asthma 0-74 and 40.6 \pm 0.3 days for Asthma $\geq 75$ years. All the considered peaks were above the $95 \%$ level of confidence. However, for some series there are a few clear peaks at high frequencies under this level of confidence (clear peaks of 7 and 3.5 days for MID 65-74 and of 7 days for MID $\geq 75$ ).

A typical and representative result of wavelet analysis is demonstrated by Fig. 1 which contains typical time behaviour and wavelet plots for CVD 0-64 year group. The power wavelet spectrum is plotted below the corresponding time series plot and shows the power spectrum (in levels of colour) scale/period (vertical axis) as a function of time (horizontal axis). The curved line at the bottom is called the cone of influence (CoI) and is determined by the analysis itself. Below the CoI, the wavelet can be subject to edge effects due to the use of a finite length series (Torrence and Compo, 1998). We consider only the information above the CoI in order to avoid spurious features caused by the wavelet method. A colour scale indicates if the periodicities have strong (red) or weak (blue) power and the black line contours a 95\% level of confidence for the existence of a periodicity for certain scale/period and time. On the right of the power wavelet spectrum plot (Fig. 1c), the average for the time series is depicted. This type of information is called the global wavelet spectrum which represents the total frequency content for all the time period. In this plot, the dashed line indicates a confidence of the $95 \%$ due to red noise (Torrence and Compo, 

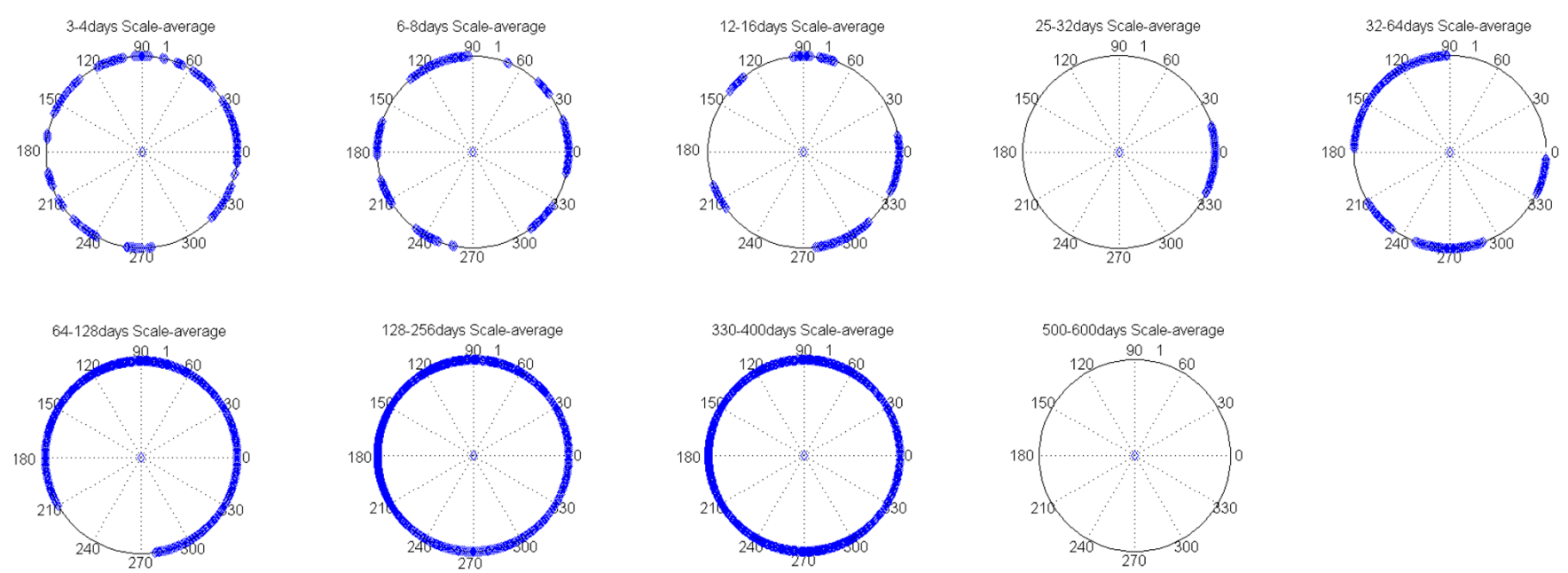

Fig. 2. Scale-average behaviour along a year (from wavelet analysis) for CVD 0-64 years group. Each plot represents a turn around the Sun. An angle of 0 degrees corresponds to 1 January of each year and every year is represented for a complete turn of 360 degrees. 90 degrees is around spring equinox, 270 degrees is around autumn equinox and 180 degrees to summer solstice.

1998). It is suggestive to perform an average along frequency bands of the power wavelet spectrum vertical axis (scale average). In this way, we are able to see specific scale/period ranges for wavelet analysis. For our study, we have arbitrarily chosen the following frequency bands: $3-4,6-8,12-16$, 25-32, 32-64, 64-128, 128-256, 330-400, 500-600, 601700 days. The result is that the appearance of $\sim 1$ year periodicity (330-400 days band) is present for all series in the wavelets within the cone of influence.

In order to analyse more carefully these frequencies and other frequency band contents, we plot the frequency content in polar graphs that represent a turn of the Earth around the Sun (see Fig. 2 as a representative example for the same data shown in Fig. 1). As the studied complete period contains 9 years ( 9 turns), the result of each plot is the average behaviour of the periodicity content around a year (a turn) beginning from the 1 January of each year and represented by angle 0 . For a rough reference guide, angles of 0, 90, 180 and 270 degrees are close to winter solstice, spring equinox, summer solstice and autumn equinox, respectively. Taking data from the right half circle (autumn-winter) as data when Earth's orbit is closer to Sun and data from the left circle (spring-summer) as data when Earth's orbit is farther away from the Sun, we are able to see the apparent distribution of periodicities for each band either close or far away from the Sun.

In relation with short period region, the presence of periodicities is higher in epochs close to the Sun for all the diseases, except for CVD groups for 6-8 days band; Asthma 0-74 for the 12-16 and 25-32 days bands and CVD 0-64 for 32-64 days band. Considering only the appearance of periodicities for time scales larger than the double or triple of the studied periodicity (e.g. for 3-4 days band, we focus on the appearance of this periodicity in time periods of at least 8 days for the two-times criterion and periods of at least 12 days for the three-times criterion) we found that regardless of the criterion, 3-4 days band content continued being always higher when Earth is closest to the Sun and occasionally it is only present during these months. The 6-8 days band has a similar behaviour except for CVD 0-64 which has an opposite result for the two times criterion. However, a stricter threetimes criterion left only one result for Asthma 75 (higher 6-8 days band content during autumn-winter time). The 12-16 days band content is higher when the Earth is closer to the Sun only for CVD 0-64 for two-times criterion. No periodicities appear for the three-times criterion. For the two-times criterion, 25-32 days band content is higher during autumnwinter time for Asthma groups but for the stricter threetimes criterion this behaviour was reversed for Asthma 064 and not present any more for Asthma 75. We found that only the two-times criterion gives a result for 32-64 and 64128 days band content being always greater during autumnwinter time for Asthma 0-74 and Asthma 75, respectively. Finally, applying the two and three-times criteria to 128-256 and 330-400 days bands, we obtained more or less the same content regardless of Earth's position around the Sun.

\section{Medical diseases and heliogeophysical proxies}

As for the medical data, we performed an FFT analysis for several heliogeophysical proxies. For sunspot numbers, there are peaks of low frequencies corresponding to $\sim 1.9 \pm$ 0.5 years, $1.02 \pm 0.06$ years, and some harmonics for one year and high frequencies corresponding to e.g. $26.6 \pm 0.9$ and $21 \pm 0.2$ days. For AA there are peaks of $1.6 \pm 151$ years, $0.9 \pm 0.06$ year, harmonics of them and in the high frequency regions, there are peaks of $27.7 \pm 0.24$ days and 

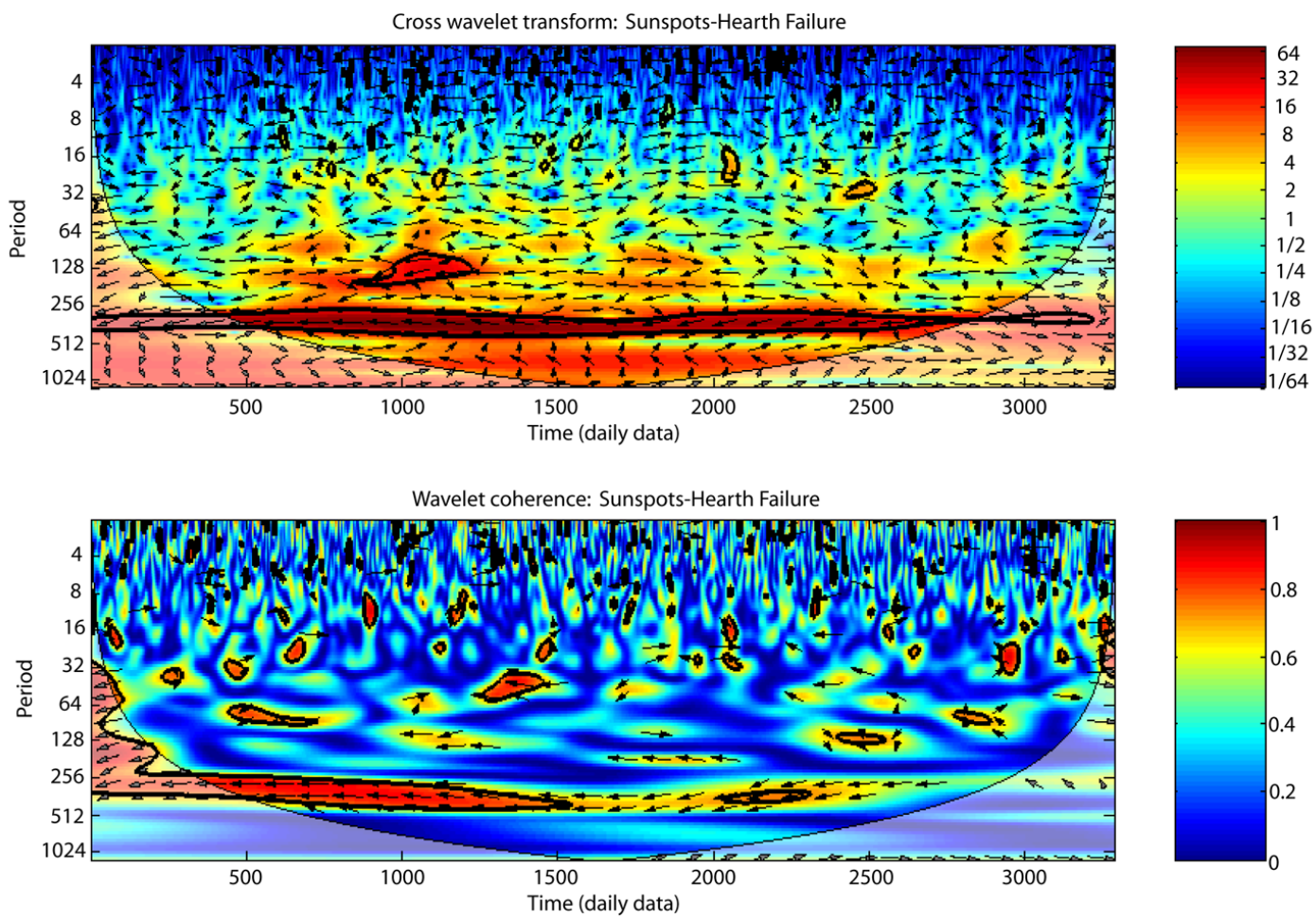

Fig. 3. Cross wavelet and wavelet coherence analysis of sunspots and HF group.

harmonics of these frequencies $(13.5 \pm 0.2$ and $6.96 \pm 0.02$ days) among others. For the Ap-index, there is also the presence of peaks for $1.6 \pm 0.3$ and $1 \pm 0.08$ year, their harmonics, $27.7 \pm 0.14$ days and its harmonics $(13.5 \pm 0.07$, $6.96 \pm 0.03,3.65 \pm 0.005$ days) among others. Periodicities close to 1 year and half year have been reported in the literature previously. A $\sim 1.3$ year periodicity has been reported in helioseismical data (Krikova and Solanki, 2002) and a lower periodicity of $154-158$ days period (about 0.43 years), known as Rieger periodicity, has been found in connection to solar flares (Rieger et al., 1984; Krikova and Solanki, 2002; Bouwer, 1992). The 27-28 day periodicity is associated with solar rotation (Lawrence et al., 2008) and $\sim 13.5$ day periodicity has been related with opposite sources activity per solar rotation and the structure of interplanetary magnetic field (Fenimore et al., 1978; Pap et al., 1990; Gonzalez et al., 1993). About 7 and 3.5 days periodicity could be harmonics of $\sim 13.5$ days although they only appear in AA and Ap-indices and not in sunspots. They have also been reported in relation to solar activity and human health (Breus et al., 2008, and references therein).

In order to search for statistically relevant correlation between the periodicities found in medical data and heliogeophysical data, we performed a cross wavelet and wavelet coherence analyses to pairs of medical and heliogeophysical series. Typical results of these two analyses are shown in Fig. 3 for sunspots and HF. In both panels, the curved line in the bottom indicates the cone of influence under which the signals must be discarded. A colour level bar indicates the intensity of each signal. A contour black line determines a 95\% level of confidence for the existence of a periodicity for certain scale/period and time. The arrows in each plot indicate the phase difference between the components of the two series (Grinsted et al., 2004). A right arrow indicates an in-phase relation while a left arrow means anti-phase. An annual anti-phase relation is present in most of the series for both cross wavelet and wavelet coherence analyses. The exceptions are AA-index and CVD 65-74 and Asthma 75, respectively.

Periodicities content coincidences for all the diseases and sunspots are plotted in Fig. 4. We note that a yearly periodicity (330-400 days band) coincidence occurs during the increasing phase of Solar Cycle 23 (from the middle of 1998 to the end of 2000 and in few diseases until the beginning of 2001). Similar results were found for AA and Ap geomagnetic indices (plots not shown). For AA-index longer time scales of coincidence for the 330-400 days band correspond to MID 65-74, CVD 0-64 and Asthma 0-74 while shorter periods correspond to MID 0-64, CVD $\geq 75$ and HF groups. For the AA-index, a half year periodicity (128-256 days band) appears around 1999 for most of the diseases except for MID 0-64, MID 65-74 and Asthma $\geq 75$ years groups. For the Ap-index, the results for 330-400 days band show shorter time scales of coincidence for MID $\geq 75$ and Asthma $\geq 75$ from the end of 1998 to part of 1999 (all 1999 for Asthma $\geq 75$ ). The wavelet coincidences also present a 


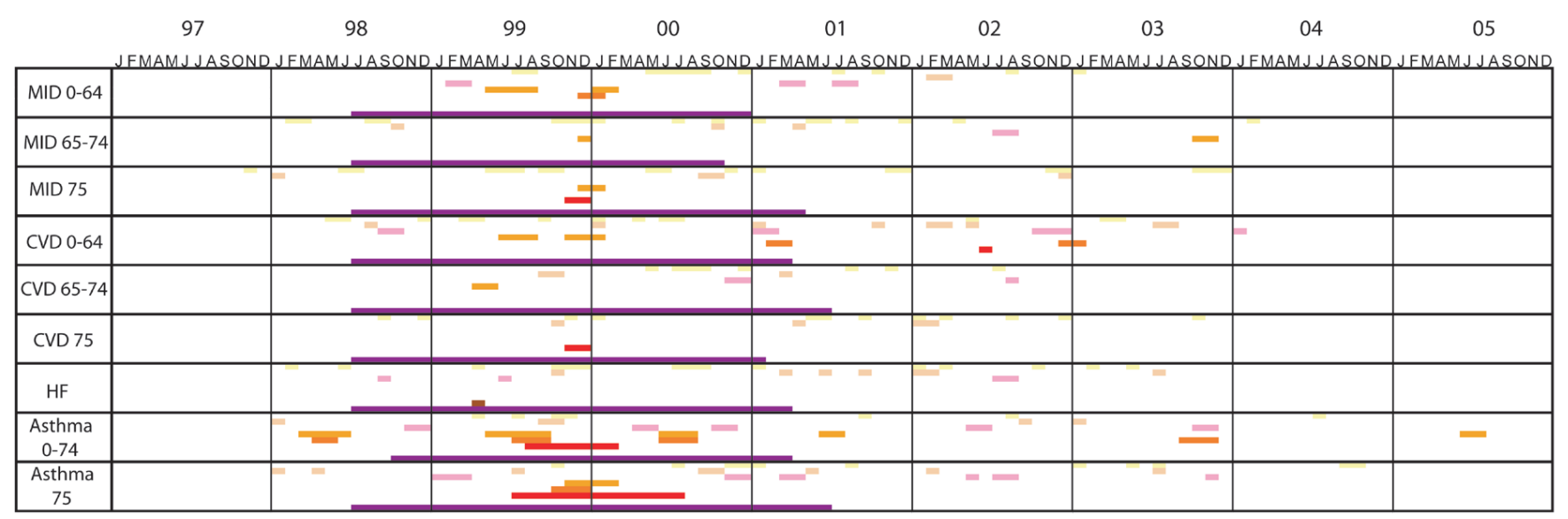

$\square_{3-4}^{30} \quad \square_{6-8} \quad \square^{12-16} \quad \square_{25-32} \quad \square_{32-64} \square_{64-128} \square_{128-256} \square_{300-400}$

Fig. 4. Cross wavelet and wavelet coherence coincident time periods by band for sunspots and all diseases.

half year periodicity (128-256 days band) around 1999 for all of the diseases except for MID 65-74 and CVD $\geq 75$. The behaviour of the high frequency content for cross wavelet and wavelet coherence coincidence periods is not so evident. However, for sunspots there are few or none coincidences during 1997, 2004 and 2005.

\section{Discussion}

Time series behaviour, FFT and wavelet analyses show that there is a presence of a predominant yearly periodicity in all time series. FFT also revealed a 1.4-1.6 year periodicity for all series except MID 0-64 and MID 65-74; an 1.5 year periodicity for $\mathrm{HF}$ and Asthma $75 ; \sim 1.8$ years for $\mathrm{HF}$; and $\sim 2.05$ years for CVD 65-74, CVD 75 and HF. Most of the series show a half year periodicity as well. This periodicity is rather interesting from a physical point of view because of the relevance in solar physics and space weather research. What the actual physical connection could be requires further detailed modelling and investigation. In the context of weather or social explanations for these periodicities, only the annual and semi-annual ones have been explained as a result of a seasonal variation for cardiac and mental diseases (Sheth et al., 1999) and asthma (Weiss, 1990) or a state of mind because of the holidays or the proximity to the end of the year (Phillips et al., 2004). Wavelet analysis reveals a frequency content of yearly and semi-yearly periodicities for all diseases as well as 601-700 days (1.64-1.92 years) only for HF and a frequency content of 500-600 days (1.371.64 years) only for Asthma 75. In wavelet analysis the yearly signal seems to be predominant over the other possible signals in the long period region. Previous results in the literature show the existence of similar periodicities in solar activity as Rieger periodicity of about 0.43 years in solar flares (Rieger et al., 1984; Krikova and Solanki, 2002; Bouwer,
$1992)$ and 1.3 years periodicity in helioseismic data (Krikova and Solanki, 2002). Medical series exhibit intermittent periodicities in the short period region for 3-4, 6-8, 25-32, 3264, 64-128 day bands as well. 6-8 days periodicity found in some diseases has often explanation in terms of a social weekly behaviour of work and rest (Spielberg et al., 1996; Wang et al., 2002). Nevertheless, the 3.5 days periodicity does not have a convincing explanation in this sense but has been found in the spectral behaviour of Kp-index and seems to be related to physiological human parameters (Breus et al., 2008). This periodicity has also been seen in the peak flux distribution of solar radio burst which produces damage to wireless communication systems (Nita et al., 2002). The $\sim 3.5$ and 7 days periodicities could be harmonics of the $\sim 13.5$ day periodicity which has been directly found in solar activity (Fenimore et al., 1978; Pap et al., 1990; Gonzalez et al., 1993). Finally, the 25-32 days band is around 27-28 day of solar rotation (Lawrence et al., 2008).

In this sense, polar plots from wavelet analysis revealed higher occurrence of short period band periodicities when the Earth is closer to the Sun around its annual orbit. The 3-4 and 6-8 days band periodic content is higher in autumnwinter time for all the diseases. For long period bands the behaviour of frequency appearance varies. Yearly periodicity (330-400 days band content) does not show any preference for times when the Earth is close or far away from the Sun. On the other hand, medical and heliogeophysical crosswavelet and wavelet coherence analysis reveal coincidences of 1-year periodicity (330-400 days band) only around solar maximum. This is a very interesting result with some important potential for further biophysical research.

For sunspots and medical diseases, a 64-128 days band also appears for Asthma groups around solar maximum, while AA/Ap and medical diseases cross wavelet and wavelet coherence analysis show the appearance of a half 
year periodicity (128-256 days band) around solar maximum for most diseases with the exception of MID groups and Asthma 75 for AA; and MID 75 and CVD 75 for Ap. An appearance of a 64-128 days band after AA/Ap maxima (May-June 2003) is shown for all groups except for MID 064, MID 65-74, CVD 0-64 for AA and Ap comparison and Asthma 75 only for AA comparison. Coincidences of long period bands around solar maximum and AA/Ap maximum show that the level of coherence measured through these two wavelet analyses is higher around high solar/geomagnetic activity.

\section{Conclusions}

In summary, our study seems to indicate a higher presence of short-period periodicities for the investigated diseases in the range of 3 days to half year during epochs when the Earth is closest to the Sun ( 3 to 8 days for the strictest criterion regarding the length of the time scale where these periodicities appear) and a high level of coherence of year and semi-year periodicities during epochs of solar and geomagnetic activity maxima.

Further studies are needed to actually assess the present results for more than one solar cycle. Also, in order to attribute the behaviour of mortality frequencies in these kinds of diseases to the Earth's orbit position around the Sun (i.e. in relevance to the Sun's electromagnetic field) and not due to seasonal changes, more analysis has to be carried out in other high-latitude countries overall or even including the Southern Hemisphere. Our results, however, must be taken into account at least at an indicative level for future more extensive studies of Sun-health relationship in the sense of searching for this relation, if any, during times of high heliogeophysical activity.

Acknowledgements. We thank the Office for National Statistics for providing us with the medical data set. R. Díaz-Sandoval acknowledges J. E. González-Gallego for all support. R. Erdélyi acknowledges M. Keray for patient encouragement and is also grateful to NSF, Hungary (OTKA, Ref. No. K83133) for financial support received.

Guest Editor M. Balikhin thanks N. Beloff and another anonymous referee for their help in evaluating this paper.

\section{References}

Babayev, E. S. and Allahverdiyeva, A. A.: Effects of geomagnetic activity variations on the physiological and psychological state of functionally healthy humans: Some results of Azerbaijani studies, Adv. Space Res., 40, 1941-1951, 2007.

Bouwer, S. D.: Periodicities of solar irradiance and solar activity indices II, Sol. Phys., 142, 365-389, 1992.

Breus, T. K., Ozheredov, V. A., Syutkina, E. V., and Rogoza, A. N.: Some aspects of the biological effects of space weather, J. Atmos. Solar-Terr Phys., 70, 436-441, 2008.
Bureau, Y. R. J. and Persinger, M. A.: Decreased latencies for limbic seizures induced in rats by lithiumpilocarpine occur when daily average geomagnetic activity exceeds 20 nanoTesla, Neurosc. Lett., 192, 142-144, 1995.

Chapman, S. and Bartels, J.: Geomagnetism Vol. 1., Oxford Press, Oxford, 1962.

Cherry, N.: Schumann Resonances, a plausible biophysical mechanism for the human health effects of Solar/Geomagnetic Activity, Nat. Hazards, 26, 279-331, 2002.

Cornélissen, G., Halberg, F., Breus, T., Syutkina, E. V., Baevsky, R., Weydahl, A., Watanabe, Y., Kuniaki, O., Siegelova, J., Fiser B., and Bakken, E. E.: Non-photic solar associations of heart rate variability and myocardial infarction, J. Atmos. Solar-Terr. Phys., 64, 707-720, 2002: Non-photic solar associations of heart rate variability and myocardial infarction, J. Atmos. Solar-Terr. Phys., 64, 707-720, 2002.

Davis Jr., G. E. and Lowell, W. E.: Solar cycles and their relationship to human disease and adaptability, Med. Hypotheses, 67, 447-461, 2006.

Díaz-Sandoval, R. and Mendoza, B.: Study of the interaction between biological cells of different shapes and sizes and electromagnetic fields produced by natural phenomena, Nat. Hazards, 31, 143-156, 2004.

Díaz-Sandoval, R., Sánchez de la Peña, S., and Chávez-Negrete, A.: Seven and 3.5-day rhythms in the incidence of myocardiopathies in Mexico, Arch. Med. Res., 39(1), 134-138, 2008.

Direnfeld, L. K.: The genesis of the EEG and its relation to electromagnetic radiation, Elec. Biol. Med., 2(2), 111-121, 1983.

Fenimore, E. E., Asbridge, J. R., Bame, S. J., Feldman, W. C., and Gosling, J. T.: The power spectrum of the solar wind speed for periods greater than 10 days, J. Geophys. Res., 83, 4353-4357, 1978.

Gonzalez, A. L. C., Gonzalez, W. D., Dutra, S. L. G., and Tsurutani, B. T.: Periodic variation in the geomagnetic activity: A study based on the Ap index, J. Geophys. Res., 98, 9215-9231, 1993.

Grinsted, A., Moore, J. C., and Jevrejeva, S.: Application of the cross wavelet transform and wavelet coherence to geophysical time series, Nonlin. Processes Geophys., 11, 561-566, doi:10.5194/npg-11-561-2004, 2004.

Healy, J. D.: Excess winter mortality in Europe: a cross country analysis identifying key risk factors, J. Epidemiol. Community Health, 57, 784-789, 2003.

Jenkins, G. M. and Watts, D. G.: Spectral analysis and its applications, Holden-Day Inc, San Francisco, p. 105, 1967.

Kane, R. P.: Did predictions of the maximum sunspot number for solar cycle 23 come true? Sol. Phys., 202, 395-406, 2001.

Kane, R. P.: Sun-Earth relation: Historical development and present status - A brief review, Adv. Space. Res., 35, 866-881, 2005.

Krikova, N. A. and Solanki, S. K.: The 1.3-year and 156-day periodicities in sunspot data: wavelet analysis suggests a common origin, A\&A, 394, 701-706, 2002.

Lawrence, J. K., Cadavid, A. C., and Ruzmaikin, A.: Rotational quasi-periodicities and the Sun heliosphere connection, Sol. Phys., 252(1), 179-193, 2008.

Mayaud, P. N.: Derivation, Meaning, and Use of Geomagnetic Indices, Geophysical Monograph 22, American Geophysical Union, Washington, p. 82-84, 1980.

Nita, G. M., Gary, D. E., Lanzerotti, L. J., and Thomson, D. J.: The peak flux distribution of solar radio bursts, Astrophys. J., 570, 
423-438, 2002.

Palmer, S. J., Rycroft, M. J., and Cermack, E. M.: Solar and geomagnetic activity, extremely low frequency magnetic and electric fields and human health at the Earth's surface, Surv. Geophys., 27, 557-595, 2006.

Panagopoulos, D. J., Karabarbounis, A., and Margaritis, L. H.: Mechanism for action of electromagnetic fields on cells, Bioch. \& Biophys. Res. Comm., 298, 95-102, 2002.

Pap, J., Tobiska, W. K., and Bouwer, S. D.: Periodicities of solar irradiance and solar activity indices I, Sol. Phys., 129, 165-189, 1990.

Phillips, D. P., Jarvinen, J. R., Abramson, I. S., and Phillips, R. R.: Cardiac mortality is higher around Christmas and New Year's than at any other time: The holidays as a risk factor for death, Circulation, 110, 3781-3788, 2004.

Press, W. H.: Numerical recipes in C: The art of scientific computing, 2nd edition, Cambridge Press, New York, 1992.

Rieger, E., Share, G. H., Forrest, D. J., Kanbach, G., Reppin, C., and Chupp, E. L.: A 154-day periodicity in the occurrence of hard solar flares?, Nature, 312, 623-625, 1984.

Sátori, G., Williams, E., and Mushtak, V.: Response of the Earthionosphere cavity resonator to the 11-year solar cycle in $\mathrm{X}$ radiation, J. Atmos. Solar-Terr. Phys., 67, 553-562, 2005.
Schlegel, K., Diendorfer, G., Thern, S., and Schmidt, M.: Thunderstorms, lightning and solar activity - Middle Europe, J. Atmos. Solar-Terr Phys., 63, 1705-1713, 2001.

Sheth, T., Nair, C., Muller, J., and Yusuf, S.: Increased winter mortality from acute myocardial infarction and stroke: The effect of age, J. Am. Coll. Cardiol, 33(7), 1916-1919, 1999.

Spielberg, C., Falkenhahn, D., Willich, S. N., Wegscheider, K., and VSller, H.: Circadian, day-of-week, and seasonal variability in myocardial infarction: Comparison between working and retired patients, Am. Heart J., 132(3), 579-585, 1996.

Torrence, C. and Compo, G. P.: A practical guide to wavelet analysis, Bull. Am. Meteorol. Soc., 79, 61-78, 1998.

Ulmer, W.: On the role of the interactions of ions with external magnetic fields in physiologic processes and their importance in chronobiology, In vivo, 16(1), 31-36, 2002.

Unal, B., Critchley, J. A., and Capewell, S.: Explaining the decline in coronary heart disease mortality in England and Wales between 1981 and 2000, Circulation, 109, 1101-1107, 2004.

Wang, H., Sekine, M., Chen, X., and Kagamimori, S.: A study of weekly and seasonal variation of stroke onset, Int. J. Biomet., 47(1), 13-20, 2002.

Weiss, K. B.: Seasonal trends in US asthma hospitalizations and mortality, JAMA, 263(17), 2323-2328, 1990. 